\title{
Cannabis use increases the risk of young people developing psychotic symptoms, particularly if already predisposed
}

Henquet C, Krabbendam L, Spauwen J, et al. Prospective cohort study of cannabis use, predisposition for psychosis, and psychotic symptoms in young people. BMJ 2004;330:11-14.

\section{Does cannabis use increase the risk of developing psychotic symptoms in young people with or without a predisposition for psychosis?}

METHODS

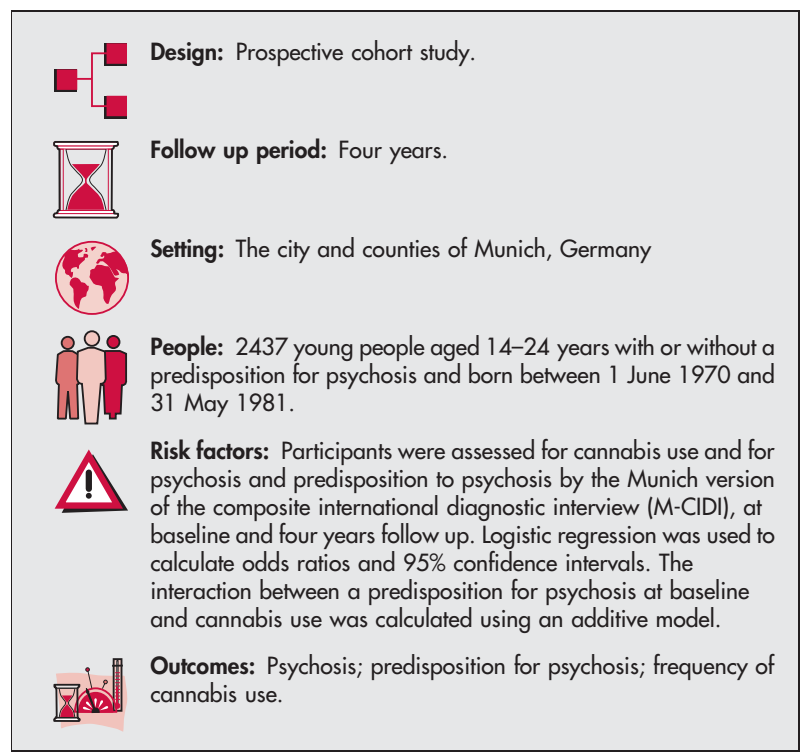

\section{MAIN RESULTS}

After adjustment for age, sex, socioeconomic status, urban residence, childhood trauma, predisposition for psychosis at baseline, and use of other drugs, tobacco, and alcohol, cannabis use increased the risk for young people developing psychotic symptoms four years later (OR: $1.67,95 \%$ CI 1.13 to 2.46 ). This effect was greater in young people with a predisposition for pyschosis at baseline compared to those without $(\mathrm{p}=0.032)$.

\section{CONCLUSIONS}

Cannabis use increases the risk for young people developing psychotic symptoms later in life, with a much greater effect in those already predisposed to pyschosis.

For correspondence: Professor Jim van Os, Department of Pyschiatry and Neuropyschology, South Limburg Mental Health Research and Teaching Networ, EURON, Maastricht University, PO BOX 616, 6200 MD Maastricht, the Netherlands

Sources of funding: grants from National Institute of Health (USA) and the German Ministry of Research, Education and Technology.

\section{NOTES}

A dose-response relation was observed between the frequency of cannabis use at baseline and the development of psychotic symptoms, which suggests causality. The authors also note previous studies which suggest that an interaction between cannabis use and a predisposition to psychosis is biologically plausible.

\section{Commentary}

I enquet et al suggest that not only does cannabis moderately increase the risk for psychosis but that it has a greater effect in those who may have a predisposition to psychosis. This recent paper makes a vital contribution to the current debate of the association between cannabis use and psychosis which is an attempt to untangle cause and effect. Do individuals start using cannabis because of some predisposition to psychosis or is cannabis implicated in the expression of psychosis?

There is research implying that cannabis use is associated with an increased risk for schizophrenia, ${ }^{1}$ supported by more recent evidence suggesting that heavy use in adolescence increases later risk of schizophrenia. ${ }^{2}$ Furthermore, this could potentially be mediated by the effects of dopamine. ${ }^{3}$ Now in the first prospective study, Henquet et al used data from a population based sample to investigate whether cannabis use at baseline increases the risk of later psychosis. These data suggest that the self-medication hypothesis is not the primary explanation for the association (although it does not entirely refute the self-medication hypothesis). The results further imply that not only does cannabis increase the risk of later psychotic symptoms but those who may be presenting with a vulnerability to psychosis are more at risk, and that there is an interaction between the early use of cannabis and a predisposition to psychosis.

Clinical implications are that cannabis can be harmful particularly for those who have a predisposition to psychosis, adding support to another area of mixed opinions - the debate as to whether cannabis should or should not be considered a harmful drug. In our practice, use of cannabis should be assessed and discouraged. Although our research evidence may not fully support the implication of cannabis in the onset of psychosis there is very little to disprove it.

Jean Addington, $\mathrm{PhD}$ Associate Professor, Department of Psychiatry, University of Toronto, Canada

1 van Os J, Bak M, Hanssen $M$, et al. Cannabis use and psychosis: a longitudinal population based study. Am J Epidemiology 2002;156:319-27.

2 Asernault L, Cannon M, Witton J, et al. Causal association between cannabis and psychosis: examination of the evidence. $\mathrm{Br} J$ Psychiatry 2004;184:110-17.

3 Caspi A, Mofitt TE, Cannon M, et al. Moderation of the effect of adolescentonset cannabis use on adult psychosis by a functional polymorphism in the catechol-O-methyltransferase gene: longitudinal evidence of a gene $\mathrm{X}$ environment interaction. Biol Psychiatry 2005;57:1117-27. 